\title{
Patient perspectives about participation in a chronic rhinosinusitis trial during the COVID pandemic: a qualitative study.
}

\author{
Jane Vennik ${ }^{1}$, Clare McDermott ${ }^{1}$, Samantha Williams ${ }^{1}$, Carl Philpott ${ }^{2}$, Mike Thomas $^{1}$, \\ Paul Little ${ }^{1}$, Anne Schilder ${ }^{3}$, and Claire Hopkins ${ }^{4}$ \\ ${ }^{1}$ University of Southampton Faculty of Medicine \\ ${ }^{2}$ University of East Anglia \\ ${ }^{3}$ evidENT, Ear Institute, University College London, UCLH Royal National Throat, Nose \\ and Ear Hospital \\ ${ }^{4}$ Guy's and St Thomas' Hospitals NHS Trust
}

May 4, 2021

\begin{abstract}
* Telephone interviews were conducted with 19 MACRO trial participants from 5 ENT sites across the UK. * Trial participants experienced mixed levels of communication during the COVID period and some felt uninformed about the trial status and their clinical situation. * Participants were most concerned about getting COVID through interactions with other patients in hospital settings. Conversely, there was a high level of trust in healthcare professionals. * Pre-visit COVID-safety information, minimising contact with other patients, and strict waiting room management can facilitate the restart of the MACRO trial from the patient perspective. * Patient confidence in trial participation is likely to continue improving with COVID vaccination roll out.
\end{abstract}

Patient perspectives about participation in a chronic rhinosinusitis trial during the COVID pandemic: a qualitative study.

Key points

- Telephone interviews were conducted with 19 MACRO trial participants from 5 ENT sites across the UK.

- Trial participants experienced mixed levels of communication during the COVID period and some felt uninformed about the trial status and their clinical situation.

- Participants were most concerned about getting COVID through interactions with other patients in hospital settings. Conversely, there was a high level of trust in healthcare professionals.

- Pre-visit COVID-safety information, minimising contact with other patients, and strict waiting room management can facilitate the restart of the MACRO trial from the patient perspective.

- Patient confidence in trial participation is likely to continue improving with COVID vaccination roll out.

\section{Introduction}

The NIHR-funded MACRO trial has been designed to evaluate endoscopic sinus surgery (ESS) and clarithromycin in the management of patients with chronic rhinosinusitis (CRS) ${ }^{1}$. The trial opened to recruitment in 2018 and by March 2020 was actively recruiting at 15 ENT sites across the UK. The onset of the COVID pandemic brought about considerable changes to clinical services and NIHR prioritisation of 
COVID-19 research studies. Consequently, MACRO trial recruitment was paused. In May 2020, the NIHR published a framework to support the restart of research activities ${ }^{2}$. Preconditions for restarting paused studies included careful consideration of research viability, research safety, and site capacity/readiness.

As part of getting ready to restart the MACRO trial, we recognised the importance of exploring the CRS patient perspective, with particular focus on their priorities, concerns and expectations for taking part in a clinical trial during the COVID period, and to identify strategies to minimise any potential anxieties around COVID safety.

\section{Methods}

We purposefully sampled 19 trial participants from 5 ENT recruitment sites in England to take part in a telephone interview. Participants had given consent to interview as part of the MACRO trial which received ethical approval from the North East - Newcastle \& North Tyneside 2 Research Ethics Committee on 20th September 2018 (REC ref.: 18/NE/0210) ${ }^{3}$. Participants were contacted by email or telephone by a researcher to arrange a suitable time for interview. We included participants with a range of characteristics including age, gender, treatment group (ESS, clarithromycin, placebo), +/-nasal polyps and prior ESS. A topic guide was used to structure the interviews but also allowed exploration of particular topics that were important to the participants. We asked questions about their current views of CRS treatments, concerns about COVID, and potential barriers and facilitators to participating in the trial. The interviews were conducted between Nov 2000 and Feb 2021 by two experienced qualitative researchers (CM and SW) who were unknown to the participants. The average length of interview was 25 minutes. All interviews were audio-recorded and transcribed verbatim, and field notes captured contextual information. Data was managed using NVivo qualitative data analysis software ${ }^{4}$. We conducted a qualitative thematic analysis ${ }^{5}$ of the interview data (a method of detailed coding of the interview transcripts, and identifying themes through refining codes and discussing within the research team). We report the findings according to the COREQ (Consolidated criteria for Reporting Qualitative research) checklist ${ }^{6}$.

\section{Findings}

Table 1 presents the characteristics of the participants who took part in the study.

The findings of the study are presented in three themes: i) experiences of trial participation, ii) safety concerns, iii) facilitating the restart of the MACRO trial.

\section{Experiences of trial participation during the COVID pandemic}

Participants' reported mixed experiences of trial participation during the first 6 months of the pandemic. Some reported feeling well-supported by the clinical team, with good communication about the trial, their future care and how to contact the team if needed. "I can't praise the process enough. I would certainly do another trial, and I wouldn't think about it at all. (Participant 12, female, medical arm)

In contrast, other participants felt communication paused once the pandemic started, reporting that they felt confused or unclear about key aspects of the trial, and the management of their CRS. This included uncertainty about whether they had finished the trial; whether they were to expect further treatment, and whether to report any new medical problems or adverse events to the trial team.

"I realise that things are difficult obviously at the moment, and so that wouldn't necessarily be seen by them as being a huge priority. On the other hand, what is the concluding step in the research? (Participant 6, male, surgical arm)

Most participants were understanding about the delays and reduced communication. However, participants who had experienced a return of their CRS symptoms or who were uncertain about their follow on treatment were particularly eager for communication to resume. 
"I've a significant improvement, generally very happy, but would like a few more answers as to why I might have experienced a second loss of senses. (participant 13, male, surgical arm)

\section{COVID safety concerns}

Whilst a third of the participants interviewed expressed no concern about participating in a trial during the COVID pandemic, the others described being unwilling to participate until the COVID risks reduced, or would need to weigh up the risks versus potential benefits.

Participants were most concerned about possible exposure to COVID from mixing with other members of the public, especially in the main hospital entrances and foyer, waiting areas and toilet areas. Participants expressed minimal confidence that other members of the public would adhere to social distancing or other COVID safety precautions.

"I think the amount of people [.....] that have been coming and going through the outpatients, well you're sort of upping your chances of catching something". (Participant 19, male, medical arm)

In contrast, participants expressed minimal concern about face to face contact with doctors or nurses, attributing this to trusting that health professionals would act properly in order to keep them safe.

"I don't I think these people of years and years of experience and training, that they're going to put themselves or me in any harm for the purposes of a trial unless they know it's perfectly safe." (Participant 1, male, medical arm)

For the majority of participants, travel was not seen as a concern for COVID safety since most travelled in their own car. Among those who used public transport, there was relatively little anxiety about COVID risks with most participants feeling that they had their own strategies for travelling.

\section{Facilitating restart of the MACRO trial}

To increase patient confidence to take part in the MACRO trial, participants wanted reassurance that COVID precautions were being taken to minimise risk. Entrances to the hospital were of particular concern, and many participants questioned the possibility of having research appointments in locations where main hospital entrances or busy public areas could be avoided.

"It's about numbers in the hospital at any given time and keeping people separated as much as possible." (Participant 3, female, medical arm)

Some participants would find pre-visit information about the precautions being taken at the hospital to be reassuring, whilst those who did not feel the need for additional information recognised the potential value for anxious patients.

"I would like the paperwork to say this is what they're doing, you know, this is what they're doing to make sure I'm safe, I don't catch anything." (Participant 8, female, medical arm)

COVID vaccination was widely perceived as reducing infection risks and increased confidence in taking part in a clinical trial. All participants reported that they would accept the COVID vaccine as soon as it was offered (several had already received their first dose) and none appeared to be anxious or sceptical about the vaccine.

I suppose it would make you feel safer from having the vaccine in your - thinking about it, it would. Yes, it probably would - yes, it would make me feel safer, yes (Participant 17, male, medical arm)

\section{Discussion}

ENT triallists faced considerable challenges in Spring 2020 at the outset of the COVID pandemic. Many routine clinical services were suspended and non-COVID research was paused to new recruits. However, 
ongoing MACRO research participants continued in the study, and sites adapted as best they could during this challenging period. Participants described a lot of understanding, but many felt uninformed about the status of the trial and their clinical situation, even 6-9 months later. As a trial team we have since put procedures in place to ensure that trial participants are better informed, both from a clinical and research perspective, in case of future interruptions to NHS services.

Our experience from talking to these research participants are that if the safety concerns could be alleviated through provision of pre-visit information, excellent waiting room management and consideration about minimising contact with the wider public during trial visits. With this in place, there are minimal barriers to restarting recruitment to the MACRO trial from a patient perspective as soon as restrictions allow, and ENT regains capacity to do so.

A key strength of this study was use of qualitative methods permitted exploration of CRS patient perspectives during the COVID pandemic and provide a valuable insight into potential barriers to trial recruitment. However, our sample was drawn from 5 of the 15 ENT sites, and perspectives may have differed at the other trial sites.

\section{Conclusion}

CRS patients are willing to consider trial participation if safety concerns are addressed. Other triallists should be confident to approach patients about taking part in a clinical trial, especially as the vaccine roll-out continues and COVID confidence improves.

1. Philpott C, le Conte S, Beard D, et al. Clarithromycin and endoscopic sinus surgery for adults with chronic rhinosinusitis with and without nasal polyps: study protocol for the MACRO randomised controlled trial. Trials 2019;20(1):246.

2. Peerdeman KJ, Hinnen C, van Vliet LM, et al. Pre-consultation information about one's physician can affect trust and treatment outcome expectations. Patient education and counseling2021;104(2):427-31.

3. McDermott C, Vennik J, Philpott C, et al. Maximising recruitment to a randomised controlled trial for chronic rhinosinusitis using qualitative research methods: the MACRO conversation study. Trials2021;22(1):54.

4. NVivo qualitative data analysis software; QSR International Pty Ltd. Version NVivo windows 2020.

5. Braun V, Clarke V. Using thematic analysis in psychology.Qualitative Research in Psychology 2006;3(2):77-101.

6. Booth A, Hannes K, Harden A, et al. COREQ (Consolidated Criteria for Reporting Qualitative Studies) Guidelines for Reporting Health Research: A User's Manual ; 2014 p214-26.

\section{List of tables}

\begin{tabular}{ll}
\hline Characteristics & $\mathbf{N = 1 9}$ \\
\hline Age Group (years) & $20-29$ (1) 30-39 (1) 40-49 (1) 50-59 (9) 60-69 (3) \\
& $70-79(4)$ \\
Sex & Male (13) Female (6) \\
Ethnicity & White British (19) \\
Previous Endoscopic Sinus Surgery & Yes (8) No (5) Surgery as part of the MACRO trial \\
& $(6)$ \\
Nasal polyps & Yes (17) No (2) \\
Randomisation & Medical Component (13) Surgical Component (6) \\
\hline
\end{tabular}




\section{Table 1 Participant characteristics}

Conflicts of interest

The authors declare that they have no competing interests.

\section{Hosted file}

Table 1.pdf available at https://authorea.com/users/411839/articles/520740-patientperspectives-about-participation-in-a-chronic-rhinosinusitis-trial-during-the-covidpandemic-a-qualitative-study 